\title{
Non-Homogeneous Poisson Process to Model Seasonal Events: Application to the Health Diseases
}

\author{
María Victoria Cifuentes-Amado* and Edilberto Cepeda-Cuervo*
}

\author{
Universidad Nacional de Colombia, Bogotá-Colombia
}

\begin{abstract}
The daily number of hospital admissions due to mosquito-borne diseases can vary greatly. This variability can be explained by different factors such as season of the year, temperature and pollution levels, among others. In this paper, we propose a new class of non-homogeneous Poisson processes which incorporates seasonality factors to more realistically fit data related to rare events, and in particular we show how the modifications applied to the special NHPP intensity function improve the analysis and fit of daily hospital admissions, due to dengue in Ribeirão Preto, São Paulo state, Brazil.
\end{abstract}

Keywords: Hospital admissions, seasonal disease behavior, non-homogeneous Poisson processes, dengue infection, cyclical process.

\section{INTRODUCTION}

Non-homogeneous Poisson processes (NHPPs) have become an important alternate tool to homogeneous processes. In fact, in many cases they are considered more realistic than the classic ones to model different day-to-day random phenomena. This is due, in part, to the fact they consider the intensity function as time-dependent. The Cox process stands out as an extension of NHPP. It is characterized by the fact that its intensity function can itself be a stochastic process. The Cox process, also known as the doubly stochastic Poisson process or mixed Poisson process has been applied in various areas such as meteorology to data on Atlantic hurricanes affecting the United States [1], neural computation to model generation of spike trains with controlled auto-and-cross-correlation functions [2], and financial mathematics [3], to estimate return on risky securities through the same issues present when modeling the structure of interest rates.

Particularly, this article deals with the daily number of admissions to public hospitals in Ribeirão Preto, São Paulo state, Brazil, and the large variations due to several factors: season of the year, climatic changes, variation in levels of different pollutants, among many others. Modeling these daily numbers is of great interest to public health administrators to avoid problems in the hospitals such as shortage of beds, equipment, drugs and health professionals.

The variation in the number of hospital admissions due to different causes has been modeled through various methods (see for example, [4-6]).

*Address correspondence to these authors at the Universidad Nacional de Colombia, Bogotá-Colombia; Tel: (+571) 3165000; Fax: (+571) 3165000 Ext. 13210; E-mail: mvcifuentesa@unal.edu.co, ecepedac@unal.edu.co

E-ISSN: 1929-6029/15
Among the various diseases that lead to hospitalization, are malaria and dengue, which are very common in public hospitals in Ribeirão Preto.

The data for the current study were provided by the Hospital Data Processing Center (PCHR, 2010) of the Department of Social Medicine, Ribeirão Preto School of Medicine, University of São Paulo. We analyzed the period ranging from January 1, 1998 to December 31, 2007. This database was composed of variables characterizing each patient's sex, age, occupation and city of residence, and variables characterizing the hospitalization as the time of entry and exit, exit condition, and the city where the disease was diagnosed and the patient was hospitalized namely.

According to [7] the dataset covers a total of 25 cities and 80,967 hospitalizations due to diseases (such as malaria and dengue), with an average of 22.4 admissions per day and median of 22 . The threshold for overcrowding corresponds to 28 hospital admissions or more in a day. Figure 1 shows the distribution of all daily admissions between January 1 , 1998 and November 31, 2007.

Our main goal is to propose seasonal count models of hospital admissions due to dengue fever using nonhomogeneous Poisson processes with different intensity functions. Different intensity functions are considered and inferences for the proposed models are obtained under the Bayesian paradigm and using the standard MCMC (Markov chain Monte Carlo) method.

The paper is organized as follows: in Section 2 we introduce some special cases of NHPP intensity functions and their uses (MMOP, GOP, etc); in Section 3 we propose the intensity and mean value functions of 


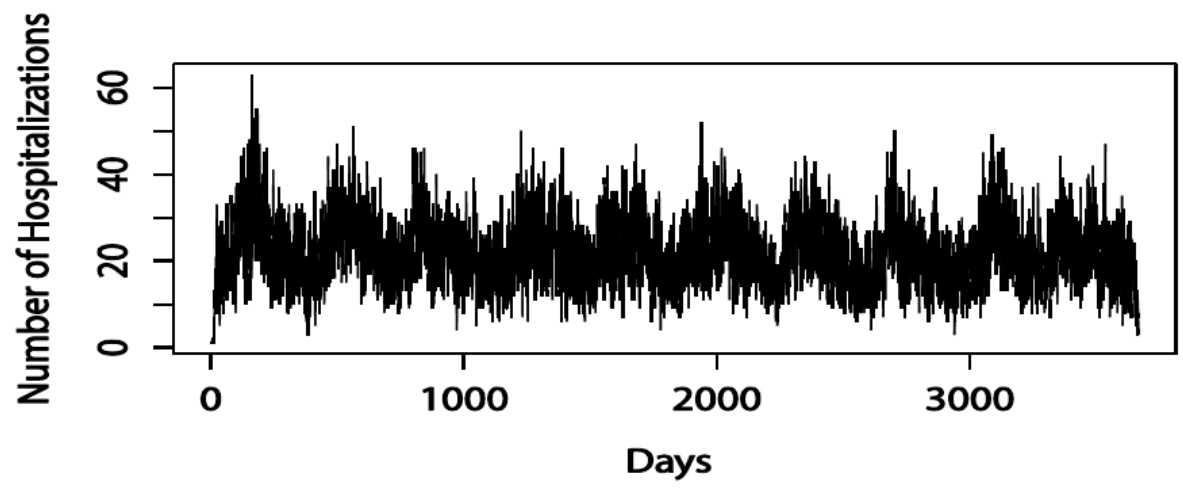

Figure 1: Daily number of dengue cases.

the new non-homogeneous Poisson process for seasonal events; Section 4 contains some comments about the likelihood function and its usefulness in Bayesian parameter estimation; Section 5 contains the application of NHPP to data of Riberão Preto and shows the fit of non-homogeneous Poisson process superposition to these cases, particularly the behavior of the new NHPP proposed in Section 4 (with seasonality included); and Section 6 summarizes our conclusions regarding the advantage of using a seasonal NHPP model to count epidemic cases.

\section{NHPP: SOME INTENSITY FUNCTIONS}

Let $\{N t: t \leq 0\}$ be a continuous process through time such that the basic NHPP properties are satisfied:

- $\quad N_{0}=0$.

- $\left.\quad P\left(N_{t+h}-N_{t}\right)=1\right)=\lambda(t) h+o(h)$

- $\quad P\left(N_{t+h}-N_{t}>1\right)=o(h)$

- Non-overlapping increments are independent.

- $\quad \lambda(t \mid \theta)=\frac{d}{d t} E\left\{N_{t}\right\}$

- $\quad \lambda_{a, b}=\int_{a}^{b} \lambda(t) d t$

- $\quad P[(N(b)-N(a))=k]=\frac{e^{-\lambda_{a b}}\left(\lambda_{a, b}\right) k}{k !}, k=0,1, \ldots$

where $t \in\{t: t \leq T\},[0, T]$ is the observed interval, $\lambda(t \mid \theta)$ is the intensity function, $\theta$ is a possibly unknown vector of parameters and $o(h)$ is a function such that, in little-o notation, satisfies $\lim _{h \rightarrow 0} \frac{o(h)}{h}=\lim _{h \rightarrow 0} \frac{P(N(t+h)-N(t)>1)}{h}=0$.
Different variations of this notation have been applied to the intensity function $\lambda(t \mid \theta)$ to model many phenomena through new approaches. Some of the special cases of NHPP, which are used in fields such as software reliability, are:

1. Power-law-process (PLP), whose expected number of failures in the first $t$ hours is $m(t)=\left(\frac{t}{\beta}\right)^{\alpha}$, for $\alpha, \beta>0$, so its intensity function is:

$\lambda^{P L P}(t)=\left(\frac{\alpha}{\beta}\right)\left(\frac{t}{\beta}\right)^{\alpha-1}: \alpha, \beta>0$

It is the model most commonly used to analyze data from a repairable system.

MOP or Musa-Okomoto process [8], which is used when failure detection is done according to a nonuniform operation profile. In this testing, the faster the defects are detected, the better the result is. The expected number of failures at time $t$ is:

$E(t \mid \theta=(\alpha, \beta))=\beta \log \left(1+\frac{t}{\alpha}\right)$ for $\quad \alpha, \beta>0$

Therefore the respective intensity function is:

$\lambda^{(M O P)}(t \mid \theta)=\left(\frac{\beta}{t+\alpha}\right): \alpha, \beta>0$.

GOP or Goel-Okumoto process. In this case we have that the failure intensity function is proportional to the residual fault content. So that:

$E(t \mid \theta=(\alpha, \beta))=\beta\left(1-e^{-\beta t}\right) \quad$ for $\quad \alpha, \beta>0$

Therefore the respective intensity function is:

$\lambda^{(G O P)}(t \mid \theta)=(\alpha \beta) e^{-\beta t}: \alpha, \beta>0$. 
GGOP or generalized Goel-Okumoto process, which represents the often found situation that the failure rate initially increases up to a time $t_{0}$, and then decreases. For this process, the expected number of failures is given by:

$$
E(t \mid \theta=(\alpha, \beta, \gamma))=\beta\left(1-e^{-\beta t \gamma}\right) \quad \text { for } \quad \alpha, \beta, \gamma>0
$$

And the respective intensity function is:

$$
\lambda^{(G O P)}(t \mid \theta)=(-\alpha \beta \gamma)\left(t^{\gamma-1}\right) e^{-\beta t}: \alpha, \beta, \gamma>0 .
$$

In the next subsections, we mention relevant research accomplished related to malaria and dengue prevention and reliable occurrence forecasts, so as to reduce the chance of death caused by collapses of health services. The mentioned methods that have been used for this purpose are highly varied, so we focus on the NHPP approach.

Let $N^{\Theta}=\left\{N_{t}: t M \in[0, T]\right\}$ be a non-homogeneous Poisson process with mean value functio $m(t \mid \boldsymbol{\theta})$, where $\boldsymbol{\theta}$ is a vector of parameters. The function $m(t \mid \boldsymbol{\theta})$ represents the expected number of events registered by $N^{(\Theta)}$ up to time $t$ (number of days with hospital overcrowding when the threshold is 28 admissions).

Equivalently, the process can be specified by its intensity function

$$
\lambda(t \mid \Theta)=\frac{d}{d t} m(t \mid \Theta)
$$

In this paper we explore some special cases of NHPP to analyze the hospital admissions data due to dengue disease of Riberão Preto: the power law process (PLP); Musa-Okumoto process (MOP) [8]; Goel-Okumoto process (GOP) [9]; and a generalized form of the Goel-Okumoto (GGOP) process.

Some relevant observations of these four special NHPP cases are:

1. The intensity function, given by the derivative of the expected value function $m(t)$, defines the hazard rate of the time between occurrence of events in the respective models.

2. The intensity function $\lambda^{(P L P)}(t \mid \Theta)$ gives the different behavior for the PLP (power law process) depending on the value of $\alpha$. As a function of time, that intensity function can be constant, decreasing or increasing depending on whether $\alpha=1, \alpha<1$ or $\alpha>1$, respectively. The intensities $\lambda^{(M O P)}(t \mid \Theta)$ and $\lambda^{(G O P)}(t \mid \Theta)$ present decreasing behavior as functions of $t$; and $\lambda^{(G G O P)}(t \mid \Theta)$ describes the situation where the intensity increases slightly at the beginning and then starts to decrease from $t$ onward.

\section{A NEW NON-HOMOGENEOUS POISSON PROCESS}

Once PLP, MOP, GOP and GGOP processes are introduced, it is possible to build new nonhomogeneous Poisson process, by superposition or addition of new terms, for example.

In the present section, we introduce some Poisson processes functions based on variations and overlap of MOP and GOP to measure their adjustment to the Ribeirão Preto data set.

First of all, we introduce the mean value function, denoted by $m(t)$ :

$$
m(t)=\beta_{0} \log \left(1+\frac{t}{\alpha_{0}}\right)+\alpha\left(1-e^{-\beta t}\right)[1+\sin (2 \pi f t+\delta)]+2 \pi \alpha f t
$$

As can be observed, this mean value function emerges from a superposition of the MOP model and a modification of the GOP one by a cyclical term plus a term depending on $t$. The first part of this mean function can be replaced by another of the special cases of NHPP studied in Chapter 2 for future work. In the current paper, the parameters of equation (6) are determined by MOP and GOP restrictions: $\beta_{0}, \alpha_{0}>0 ; \alpha, \beta>0$ where $f$ refers to the frequency of the cyclical behavior and $\delta$ is a phase parameter. Then its intensity function is:

$$
\begin{aligned}
\lambda(t) & =\left(\frac{\beta_{0}}{t+\alpha_{0}}\right)+\alpha e^{-\beta t}[1+\sin (2 \pi f t+\delta)] \\
& +2 \pi \alpha f\left[\left(1-e^{-\beta t}\right) \cos (2 \pi f t+\delta)+1\right]
\end{aligned}
$$

To illustrate the behavior of the mean value $m(t)$ and intensity $\lambda(t)$, we assume the parameter values reported in Table 1 and under such values we plot the graph in Figure 2.

As can be observed in Figure $\mathbf{2} \mathbf{a}$, the mean value function $m(t)$ shows an increasing and staggered behavior in function of $t$, meaning that its growth is not strict, such as the behavior of hospital overcrowding: some days, there is no overloading, so during those days the accumulated days of overcrowding remains equal to previous days. For that reason, equation (6) is an appropriate function to model the mean value of the 
Table 1: Initial Parameters Values

\begin{tabular}{|c|c|c|c|c|c|c|}
\hline Parameter & $\alpha_{0}$ & $\beta_{0}$ & $\alpha$ & $\beta$ & $\delta$ & $f$ \\
\hline \hline Value & 50 & 10 & 12 & 30 & 0 & 0.00277778 \\
\hline
\end{tabular}

non-homogeneous Poisson process for counting the accumulated cases of over-hospitalizations due to dengue in Ribeirão Preto, Brazil. On the other hand, in Figure $\mathbf{2 b}$, the intensity function shows a cyclical dynamic (each about 360 days) and its positivity guarantees the non-decreasing property of $m(t)$, since $\lambda(t)=\frac{d}{d t} m(t)$.

Moreover, it is possible to observe more carefully the new process's behavior. For example, if we change the value of the parameters, one at a time, this reveals the influence of each parameter in the model (Figure $3)$.

The first part, Figure $\mathbf{3 a}$ shows three curves representing changes in $\boldsymbol{m}(\boldsymbol{t})$ for different values of $\alpha_{0}$. As can be seen from the dotted line, when $\alpha_{0}$ decreases, the values of $\boldsymbol{m}(\boldsymbol{t})$ are greater, which was expected, since $\alpha_{0}$ is the divisor in equation (6). However, change is more significant for lower values, such as $\alpha_{0}=5$, than for $\alpha_{0}=100$, because the last one (segmented-line curve) is almost the same as the initial curve, when $\alpha_{0}=50$.

In turn, in Figure $\mathbf{3 b}$ the only parameter modified is $\beta_{0}$, which affects the MOP part of the equation (6), and when it has greater values, $\boldsymbol{m}(\boldsymbol{t})$ also increases, but in a much smaller proportion. These graphs measure the influence of variations of GOP-part parameters ( $\alpha$ and

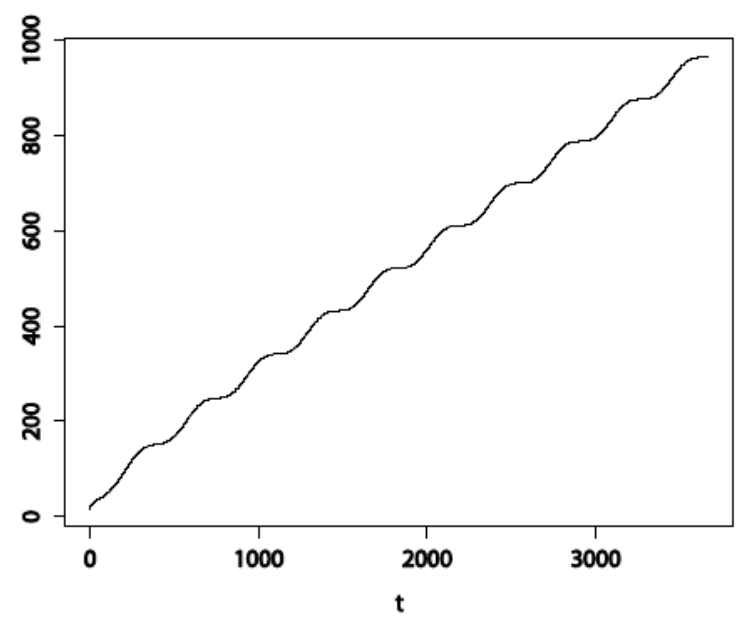

(a) Mean Value $m(t)$ $\beta$ ) on mean value function behavior, indicating that $\alpha$ is the most influential parameter for the $m(t)$ dynamic: as shown in Figure 3c, a slight increase in $\alpha$ (from $\alpha=$ 12 to $\alpha=20$ ) causes the mean value function to exhibit the largest growth in the four graphs. The reason is that the last term in the mean value function, defined in equation (6), which has the variable $t$, is multiplied by $\alpha$. Likewise, when $\alpha$ decreases to $1, m(t)$ reaches the lowest value, even if it is nearly a straight line (see dotted-line in Figure 3c. On the another hand, the GOP-part, which depends on parameter $\beta$, has the smallest influence on $m(t)$ : its graph shows that large $\beta$ values, for instance 1000 , do not significantly modify the mean value function (just a few decimal positions), in fact as $\beta>0$ then $0<(1-e-\beta t)<1$.

\section{THE LIKELIHOOD FUNCTION}

Denoting the data set by $D_{T}=\left\{n ; t_{1}, t_{2}, \ldots, t_{n} ; T\right\}$ where $n$ is the number of observed occurrences, such that $<t_{1}<t_{2}<\cdots<t_{n}<T$, where these values are the intervals of hospital over-admissions up to time $T$, the likelihood function for $\beta$ considering the time truncated model is (see for example, [10]) given by

$$
L(\theta, D t)=\left[\prod_{i=1}^{n} \lambda\left(t_{i} \mid \theta\right)\right] \exp [-m(T \mid \theta)]
$$

We can obtain inferences about the parameters of the model under a Bayesian approach. Bayesian

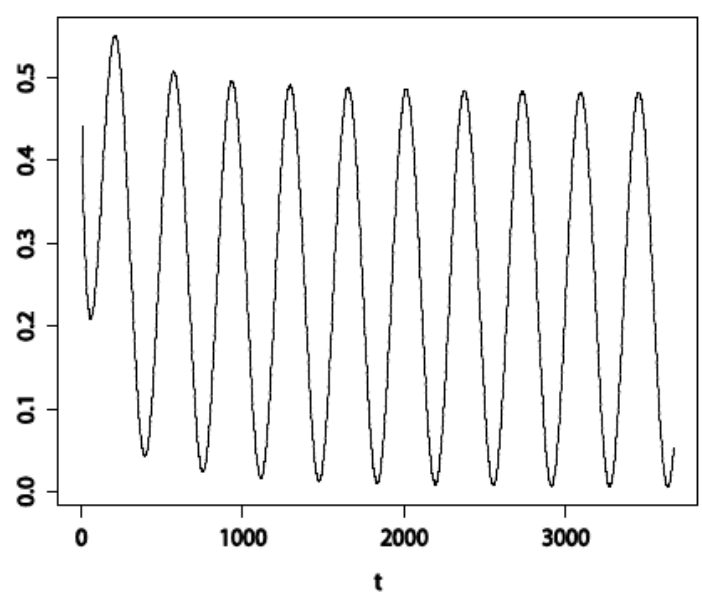

(b) Intensity Function $\lambda(t)$

Figure 2: New process mean value and intensity functions. 


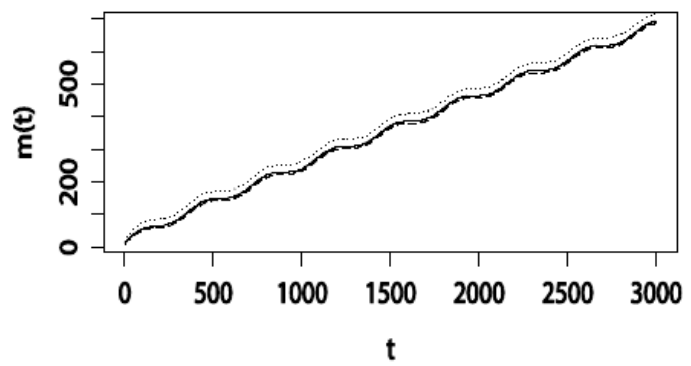

(a) $\alpha_{0}=5,50,100$

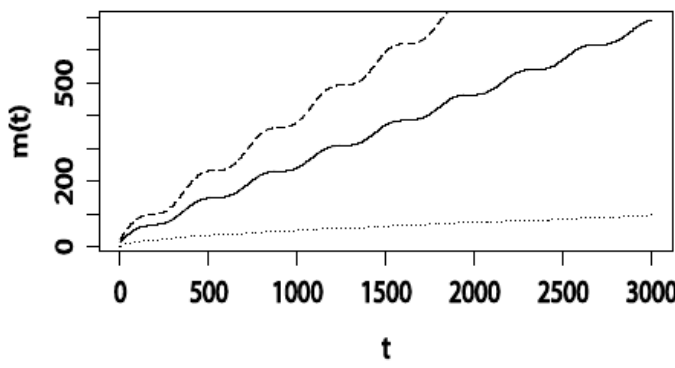

(c) $\alpha=20,12,2$

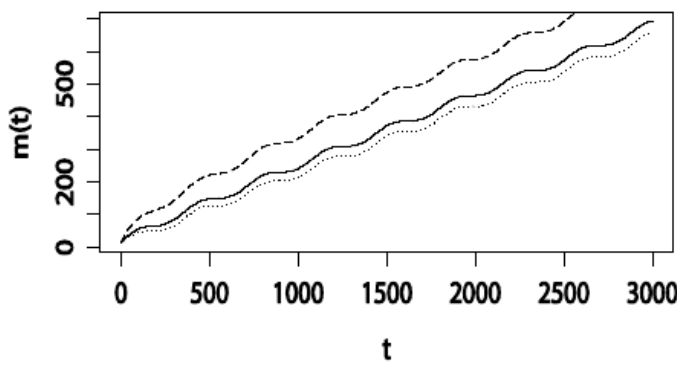

(b) $\beta_{0}=40,10,1$

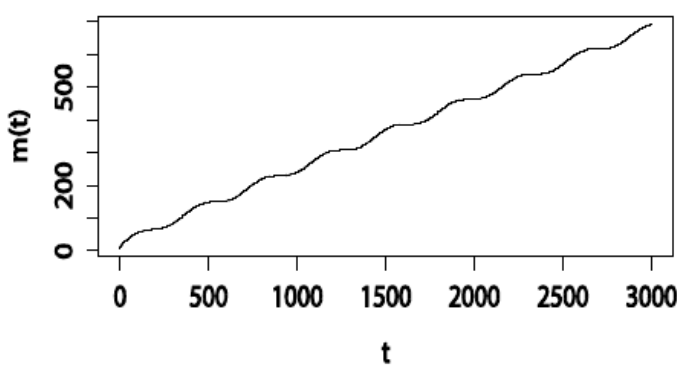

(d) $\beta=1,30,1000$

Figure 3: Sensitivity of the mean value function $\boldsymbol{m}(\boldsymbol{t})$.

inferences for NHPP have been discussed by many authors (see for example, [11-13]).

Posterior summaries of interest are obtained using standard MCMC methods (see for example, [14-16]).

Note that (8) has a slight modification in the superposition case. In [17], the authors expressed the likelihood function for the superposition Poisson process, just adding the intensity and mean value functions of each compound process:

$L(\theta, D t)=\left(\prod_{i=1}^{n} \lambda_{1}\left(x_{i}\right)+\cdots+\lambda_{J}\left(x_{i}\right)\right)$

where $\quad 0<x_{1}<\cdots<x_{n}<t, D t=\left\{x_{1}, \ldots, x_{n}\right\} \quad$ and the considered process is the superposition (sum) of $J$ processes and each one has intensity function equal to $\lambda_{i}(t)$ and mean value $m_{i}(t)$.

Later, equation (9) will be used to estimate parameters that allow adjusting superposition of the special NHPP model to the Ribeirão Preto data set.

\section{APPLICATION TO RIBEIRÃO PRETO DATA}

In this section, we analyze the daily hospital admissions count due to dengue infection in Ribeirão Preto for the period ranging from January 1, 1998 to December 30, 2007. From this dataset, we observe that 948 times there were at least 28 hospital

admissions due to dengue in the period of $T=3670$ days.

\subsection{Modeling Hospital Overcrowding}

To model the number of times that hospital overcrowding occurs in a state or city, the number of dengue cases in a population through time or the number of cases of malaria in a population, can be studied and statistically modeled by considering a point process to count these occurrences. Let $N=\left\{N_{t}: t \in(0, T]\right\}$ be the process that registers the cumulative number of daily (monthly or yearly) cases observed during the interval $(0, T)$, i.e., for each $t \in[0, T], N_{t}$ is the number of cases (hospital admission peaks, number of cases of dengue or number of cases of malaria) observed during the time interval $(0, t)$. We assume that $N$ can be modeled by a nonhomogeneous Poisson process (NHPP) with intensity function,

$\lambda(t)=\frac{d}{d t} m(t)=\frac{d}{d t} E\left(N_{t}\right)$

where $m(t)$ is the mean value function.

Figure 4 shows that the accumulated number of daily hospital admissions has increasing behavior during the observed period, taking into account that the number of dengue cases has cyclical behavior over time. 


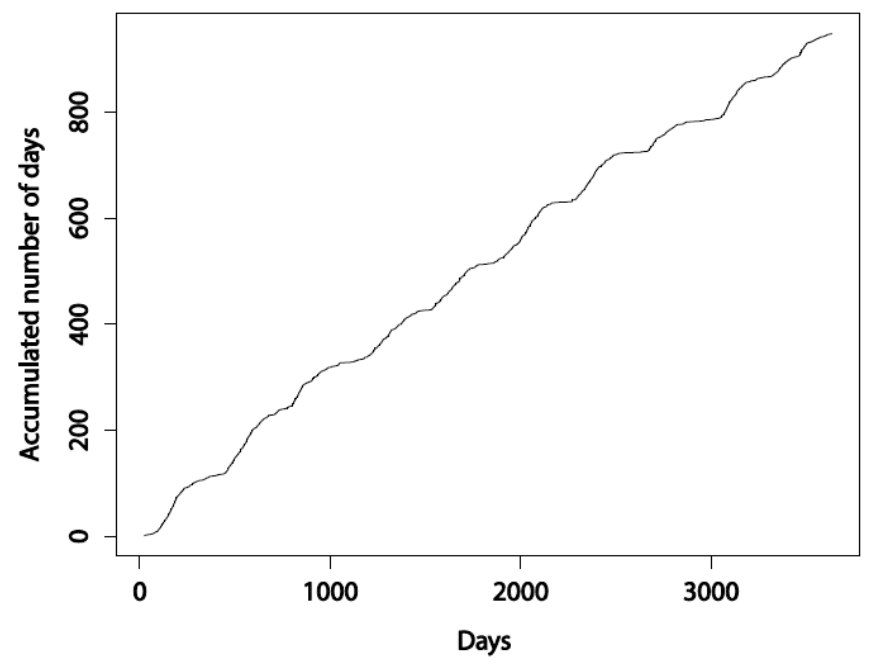

Figure 4: Accumulated daily hospital admission.

Therefore, for initial modeling of the count data, it is interesting not only to have intensity functions $\lambda(t), t \geq 0$, that are monotonically decreasing or monotonically increasing through $t$, but also that take into account the cyclical behavior of the phenomenon.

In the current paper, to analyze Ribeirão Preto count data, we apply different non-homogeneous Poisson processes, by assuming superposition of previous intensity functions, and comparing them with the new NHPP proposed in section 3.

\subsection{Parameter Estimation}

In Achcar-Cepeda-Edson (2012) [7], the four special NHPP intensity functions, reviewed in the first section, were fitted to the count of hospital over-admissions in Ribeirão Preto, Brazil in the period ranging from January 01, 1998 to December 31, 2007 and Bayesian approach was assumed to estimate each parameter.
In this simulation procedure, we used the WinBugs software [13]) to get appropriate estimates of the parameters.

The following table was obtained by [7] and has the prior distributions for each parameter and the posterior summaries accomplished by Open Bugs software for PLP, MOP, GOP and GGOP models:

To get these results, we considering a burn-insample of size 5000 and 20000 simulated samples, taking every 20th iteration, using WinBugs. The convergence was monitored using trace-plots of the Gibbs samples simulated for each parameter.

In the next sections, we explore the adjustment of different variations of NHPP functions, including the new one introduced in Figure 6. For this purpose, we use Table $\mathbf{2}$ for prior parameter distributions and in some cases, we choose the hyperparameter values to have convergence of the Gibbs sampling algorithm.

\subsection{MOP Process and Hospital Overcrowding}

In [7], the authors (Achcar, Cepeda and Zangiacomi) showed that the MOP process, among the four usual ones, best fits the hospital data. Figure $\mathbf{5}$ shows the empirical accumulated number of days of overcrowding and the fitted mean value function for the MOP process.

The parameter values which generate the fitted MOP curve are those indicated in Table 2 for the MOP function. In regard to the accuracy of the estimate, it produced a DIC value of 1900450 , which is high but not as much as the other ones produced by WinBugs for PLP, GOP and GGOP. Therefore, MOP gives better fit,

Table 2: Prior and Posterior Summaries

\begin{tabular}{|c|c|c|c|c|}
\hline Model & Parameter & Prior & Mean & S. D. \\
\hline \multirow{2}{*}{ PLP } & $\alpha$ & $\mathrm{U}(0,2)$ & 0.9232 & 0.03081 \\
\hline & $\beta$ & $\mathrm{U}(0,100)$ & 2.244 & 0.5621 \\
\hline \multirow{2}{*}{ MOP } & $\alpha$ & $U(0,10000)$ & 6964.0 & 1511.0 \\
\hline & $\beta$ & $U(0,3000)$ & 2238.0 & 399.1 \\
\hline \multirow{2}{*}{ GOP } & $\alpha$ & $U(1,10000)$ & 2818.0 & 719.0 \\
\hline & $\beta$ & $\mathrm{U}(0,1)$ & 0.0001205 & 0.0000298 \\
\hline \multirow{3}{*}{ GGOP } & $\alpha$ & $U(0,5000)$ & 2229.0 & 655.6 \\
\hline & $\beta$ & $\mathrm{U}(0,0.001)$ & 0.000104 & 0.000028 \\
\hline & $\gamma$ & $\mathrm{U}(0.5,1.5)$ & 1.058 & 0.05368 \\
\hline
\end{tabular}


but this could be improved by considering that MOP is a strictly increasing function, so the introduction of cyclical factor might improve the accuracy.

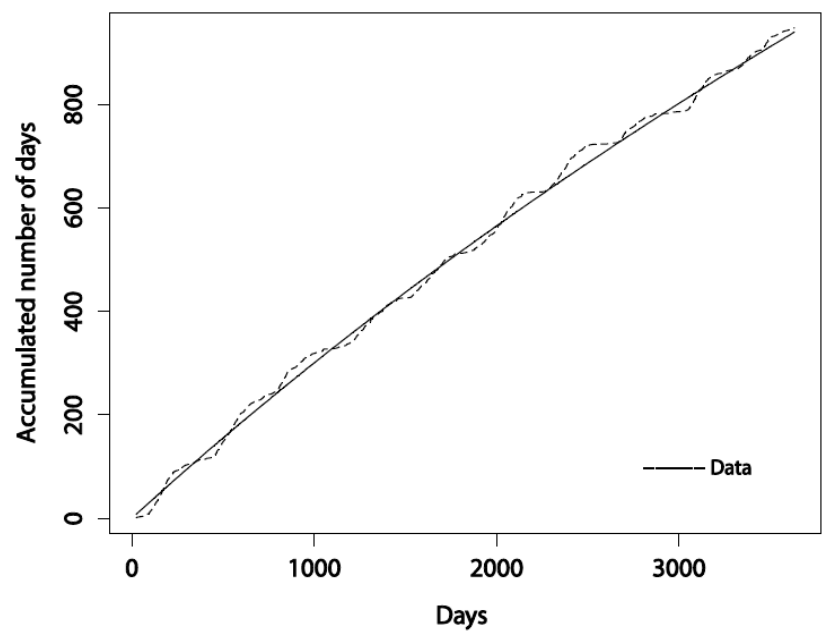

Figure 5: MOP function and accumulated days with overcrowding.

\subsection{Overlap between MOP and PLP}

As an intermediate step, in this sub section we consider the superposition of MOP and PLP in weighted form, thus:

$m(t)^{M O P+P L P}=\beta_{0} \log \left(1+\frac{t}{\alpha_{0}}\right)+\left(\frac{t}{\beta}\right)^{\alpha}$

With intensity function equal to:

$\lambda(t)^{M O P+P L P}=\frac{\beta_{0}}{t+\alpha_{0}}+\left(\frac{\alpha}{\beta}\right)\left(\frac{t}{\beta}\right)^{\alpha-1}$

Through WinBugs, after of 200000 iterations we ran the $M O P+P L P$ model and obtained the values presented in Table 3.

However, we could improve this model by adding a $p$ value in order to assign a weight to each MOP and PLP function. For this $p$, we assumed a noninformative prior distribution, that is to say, $p \sim U(0,1)$ and the results were as follows:
Comparing the DIC values, it looks like the weighted option does not offer any improvement in comparison with superposition. In fact, in Table $\mathbf{5}$ the value of $p$ shows that the model has assigned greater weight to the PLP model.

Lastly, here are the comparative graphs between the $M O P+P L P$ model and the accumulated data of hospital overcrowding.

\subsection{New NHPP and Hospital Overcrowding}

For the new NHPP introduced in (6), parameters $\alpha_{0}, \beta_{0}, \alpha$ and $\beta$ are estimated assuming the same distributions as in Achcar-Cepeda-Martinez (2012) [7] for MOP and GOP processes. Therefore the only missing parameter is $f$, the frequency that appears inside the cyclical term of the new process. For this purpose, we used the RStudio software to apply the spectral analysis tool and to get a frequency estimator.

In Figure 7, the frequency of overcrowding, $f$, is assumed to be about $(1 / 360)$ which means that each 360 days, the cycle of over-admissions starts, which is consistent with the mosquito cycle life.

On the other hand, for the new process we introduced a new parameter, $\delta$, which represents the phase of the cycle, and it depends on the first observation moment. Therefore, we can take a uniform prior distribution $U(0, \pi)$.

From the NHPP model run with WinBugs, we got the following parameter values:

For the three reviewed models, the $D I C$ was $1.9 \mathrm{E}+6$ (because of software rounding effect), but the new model captures the cyclical behavior of the data:

In order to compare the fit of the models to the data, their mean squared errors were calculated and reported in the next table:

Table 3: Prior and Posterior Summaries for Superposition of MOP+PLP

\begin{tabular}{|c|c|c|c|c|}
\hline Parameter & Prior & Mean & S. D. & Confidence Intervals 95\% \\
\hline \hline$\alpha_{0}$ & $\mathrm{U}(0,10)$ & 0.2088 & 0.02467 & $(0.2108,0.2156)$ \\
\hline$\beta_{0}$ & $\mathrm{U}(0,30)$ & 0.0033 & 0.1677 & $(1.17 \mathrm{E}-5,0.002832)$ \\
\hline$\alpha$ & $\mathrm{U}(0,2)$ & 0.67 & 0.078 & $(0.6745,0.6937)$ \\
\hline$\beta$ & $\mathrm{U}(0,10)$ & 0.1454 & 0.037 & $(0.145,0.151)$ \\
\hline
\end{tabular}


Table 4: Prior and Posterior Summaries for Weighted Overlap of MOP+PLP

\begin{tabular}{|c|c|c|c|c|}
\hline Parameter & Prior & Mean & S. D. & Confidence Intervals 95\% \\
\hline \hline$\alpha_{0}$ & $\mathrm{U}(0,10)$ & 2.187 & 0.07011 & $(1.313,1.334)$ \\
\hline$\beta_{0}$ & $\mathrm{U}(0,30)$ & 1.319 & $0.04095,2.236)$ \\
\hline$\alpha$ & $\mathrm{U}(0,2)$ & 0.9372 & 0.0153 & $(0.9268,0.9508)$ \\
\hline$\beta$ & $\mathrm{U}(0,10)$ & 2.456 & 0.1018 & $(2.468,2.562)$ \\
\hline$p$ & $\mathrm{U}(0,1)$ & 0.0001036 & 0.0015 & $(1.748 \mathrm{E}-6,7.99 \mathrm{E}-4)$ \\
\hline
\end{tabular}

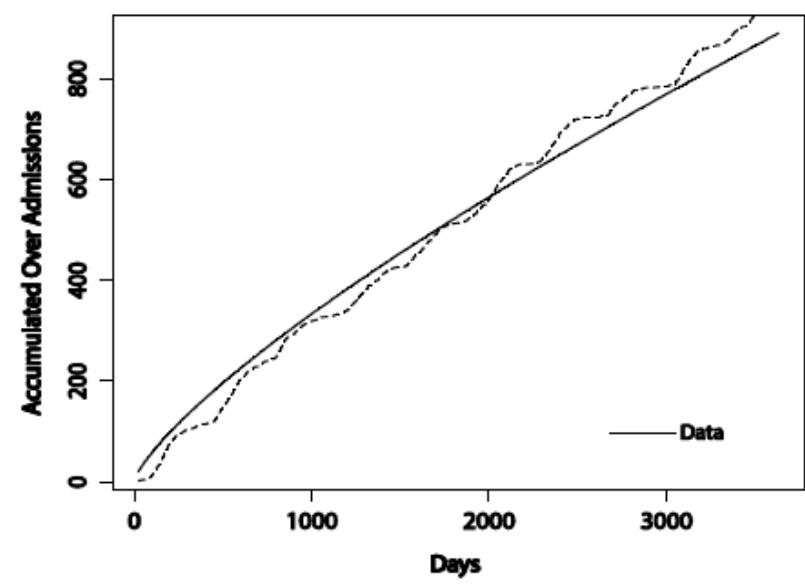

(a) Superposition MOP and PLP

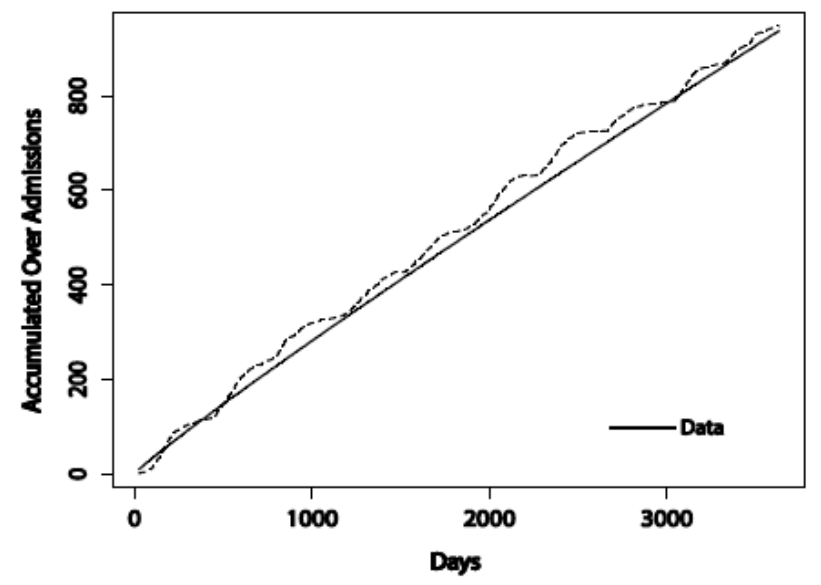

(b) Weighted Superposition MOP and PLP

Figure 6: Superposition of MOP and GOP fitted to Ribeirão Preto data.
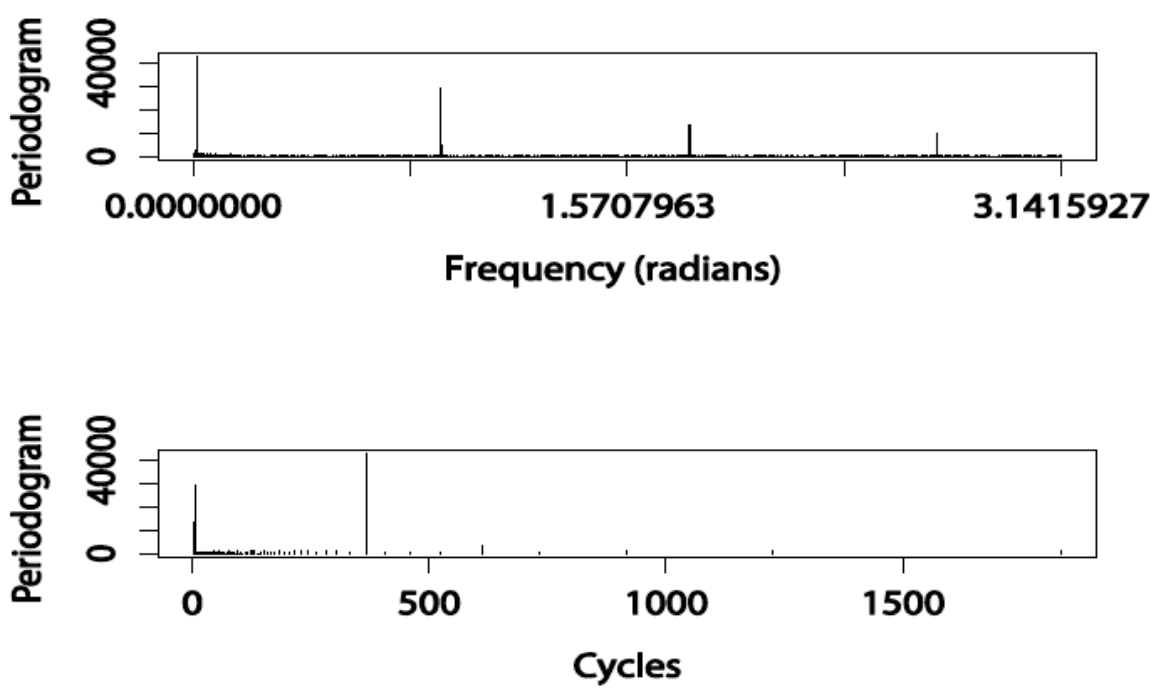

Figure 7: MOP function and accumulated days with over-admissions.

According with the last results, the new NHPP, which includes a superposition and a cyclical factor, is the model that fits the hospital overcrowding data most accurately.

\section{CONCLUDING REMARKS}

In this paper we introduced new modeling approaches to analyze count data due to hospital over- 
Table 5: Prior and Posterior Summaries for the New NHPP

\begin{tabular}{|c|c|c|c|c|}
\hline Parameter & Prior & Mean & S. D. & Confidence Intervals 95\% \\
\hline \hline$\alpha_{0}$ & $\mathrm{U}(10,90)$ & 52.998 & 9.536 & $(36.91,69.19)$ \\
\hline$\beta_{0}$ & $\mathrm{U}(0,20)$ & 19.98 & 1.548 & $(11.9,27.44)$ \\
\hline$\alpha$ & $\mathrm{U}(0,20)$ & 13.59 & 0.6729 & $(12.91,14.34)$ \\
\hline$\beta$ & $\mathrm{U}(0,10)$ & 0.001 & 2.095 & $(0.00025,4.659)$ \\
\hline$\delta$ & $\mathrm{U}(0, \pi)$ & 2.67 & 0.04961 & $(2.537,2.686)$ \\
\hline
\end{tabular}

admission, in particular, due to malaria and dengue in Ribeirão Preto, Brazil. The use of a non-homogeneous Poisson processes assuming different intensity functions gives great flexibility to fit for the count data. We also introduced a new model considering the superposition of two models plus the introduction of a cyclical term to capture the seasonality of the disease. This new modeling approach can be used to analyze data regarding many different seasonal diseases and it showed better fit to the data, with a lower DIC than the other ones studied.

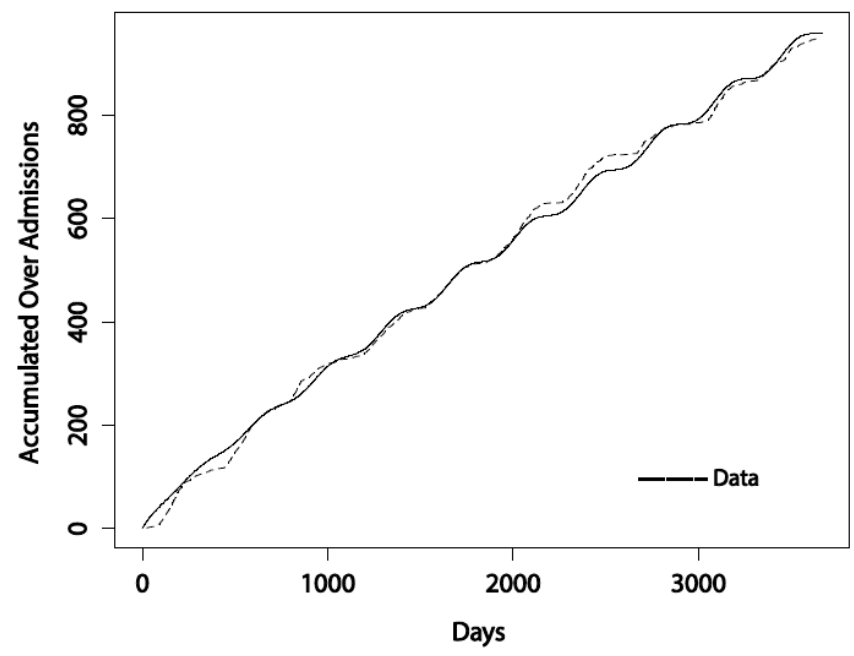

Figure 8: The new NHPP and Riberao Preto data.

Table 6: Mean Square Errors for the Reviewed Models

\begin{tabular}{|c|c|}
\hline Model & MSE \\
\hline \hline MOP+PLP & 0.849716 \\
\hline MOP+PLP Weighted & 0.474346 \\
\hline New NHPP & 0.233450 \\
\hline
\end{tabular}

The use of Bayesian methods considering standard existing MCMC simulation methods to generate samples for the joint posterior distribution of interest, especially using the WinBugs software, greatly simplifies accurate inference and accurate predictions. In that respect, it is possible to achieve a substantial advance in non-homogeneous process modeling and analysis.

\section{REFERENCES}

[1] Garrido J, Lu Y. On double periodic non-homogeneous Poisson processes. Bulletin of the Association of Swiss Actuaries 2004; 2: 195-212.

[2] Krumin M, Shoham S. Generation of spike trains with controlled auto- and cross-correlation functions. Neural Comput 2009; 21: 1642-1664 http://dx.doi.org/10.1162/neco.2009.08-08-847

[3] Lando D. On Cox processes and credit risky securities. Review of Derivatives research 1998; 2(2-3): 99-120.

[4] Müller-Pebody B, et al. Modelling hospital admissions for lower respiratory tract infections in the elderly in England. Epidemiology and Infection 2006; 134.06: 1150-1157. http://dx.doi.org/10.1017/S0950268806006376

[5] Burnett Richard T, et al. Effects of low ambient levels of ozone and sulfates on the frequency of respiratory admissions to Ontario hospitals. Environmental Research 1994; 65.2: 172-194.

http://dx.doi.org/10.1006/enrs.1994.1030

[6] Pievatolo A, Ruggeri F. Bayesian reliability analysis of complex repairable systems. Appl Stoch Models Bus Ind 2004; 20: 253-264. http://dx.doi.org/10.1002/asmb.522

[7] Achcar JA, Cepeda-Cuervo E, Martinez EZ. Use of NonHomogeneous Poisson Processes in the Modeling of Hospital over Admissions in Ribeirão Preto and Region, Brazil: An Application to Respiratory Diseases. J Biomet Biostat 2012; 3: 131 http://dx.doi.org/10.4172/2155-6180.1000131

[8] Musa J, Okumoto K. A logarithmic Poisson execution time model for software reliability measurement. Proceedings of the 7th international conference on Software engineering. IEEE Press 1984.

[9] Goel AL, Okumoto K. An analysis of recurrent software failures on a real-time control system. Proceedings of ACM Conference 1978.

[10] Cox D, Lewis P. Statistical analysis of series of events. Methuen \& Co Ltda, UK 1966.

[11] Kuo L, Yang T. Bayesian computation for nonhomogeneous Poisson process in software reliability. Journal of the American Statistical Association 1996; 91: 763-773. http://dx.doi.org/10.1080/01621459.1996.10476944

[12] Ramirez-Cid J, Achcar JA. Bayesian inference for nonhomogeneous Poisson process in software reliability models. Comput Stat Data Anal 1999; 32: 147-159. http://dx.doi.org/10.1016/S0167-9473(99)00028-6 
[13] Lunn DJ, Thomas A, Best N, Spiegelhalter D. WinBUGS - a Bayesian modelling framework: concepts, structure, and extensibility. Statistics and Computing 2000; 10: 325-337. http://dx.doi.org/10.1023/A:1008929526011

[14] Chib S, Greenberg E. Understanding the metropolis-hastings algorithm. The American Statistician 1995; 49.4: 327-335.

[15] Gelfand AE, Smith AFM. Sampling-based approaches to calculating marginal densities. Journal of the American Statistical Association 1990; 85.410: 398-409.

http://dx.doi.org/10.1080/01621459.1990.10476213
[16]

Smith AFM, Roberts GO. Bayesian computation via the Gibbs sampler and related Markov chain Monte Carlo methods. J R Stat Soc Series B Stat Methodol 1993; 3-23.

[17] Kuo L, Yang T. Bayesian computation for the superposition of nonhomogeneous Poisson process. Can J Stat 1999; 27 : 547-556.

http://dx.doi.org/10.2307/3316110

Received on 12-08-2015

http://dx.doi.org/10.6000/1929-6029.2015.04.04.4

(C) 2015 Cifuentes-Amado and Cepeda-Cuervo; Licensee Lifescience Global.

This is an open access article licensed under the terms of the Creative Commons Attribution Non-Commercial License (http://creativecommons.org/licenses/by-nc/3.0/) which permits unrestricted, non-commercial use, distribution and reproduction in any medium, provided the work is properly cited. 